\title{
A Computer Aided Automatic Classification of Eye Dryness Using Tear Ferning Characteristics
}

\author{
Ali S Saad ${ }^{* 1}$ and Ali M Masmali ${ }^{2}$ \\ ${ }^{1}$ Department of Biomedical Technology, King Saud University, Saudi Arabia \\ ${ }^{2}$ Cornea Research Chair (CRC), Department of Optometry College of Applied Medical Sciences, King Saud University
}

Received: 䟧 August 30, 2018; Published: 制 September 06, 2018

*Corresponding author: Ali S Saad, Department of Biomedical Technology, College of Applied Medical Sciences, King Saud University, P. 0. Box 10219, Riyadh 11433, Saudi Arabia

\begin{abstract}
Patients with long term dry eye (DE) could suffer cornea damage. To date, there is no universal diagnostic test to detect eye dryness. Recently, an automated diagnostic method of DE using thermographic images provided a good binary classification between normal and DE subjects. The present work focuses on the automatic diagnosis of DE patients using tear ferning (TF) images. The TF images for 100 subjects were used to classify their degree of dryness automatically (without human interaction) using a five-point grading scale. First, from each TF image a vector characteristic (VC) was computed to represent each patient during the automatic classification. Then, it was compared to the VC references representing the five points grading scale. Next, each subject's TF image was assigned to the closest reference. The classification of each patient's degree of dryness using the vector characteristic is based on a decision tree approach using "See5": tools for data mining that generates decision trees for classification purpose. The fully automatic classification (diagnosis) provides a promising result compared to the manual classification by two experts. It was found that $81 \%$ of the automatic classification perfectly matched the manual ones. $12 \%$ of the automatic grades were misclassified to the next grade (i.e. one grade difference between the automatic and manual grading). Only $7 \%$ were completely misclassified. Future work on the VC and the algorithm of classification using synthetic TF images could further improve the automatic classification of dry eye patients.
\end{abstract}

\section{Introduction}

The healthy tear film is important to keep the eye lubricated, healthy and optically [1]. The main components in tears are electrolytes (e.g. cations such as $\mathrm{Na}^{+}$and $\mathrm{K}^{+}$), water, proteins (e.g. lysozyme and lactoferrin), lipids, mucins and others [2]. Tear film contains three layers, aqueous, lipid and mucin, and its stability affects vision quality and eye health [3]. Dry eye (DE) leads to a disturbance in both tear volume and quality because of the changes in the ocular surface [4]. The most common dry eye symptoms include itchiness, grittiness, blurriness and photophobia [5] Diagnosis of dry eye is very challenging since multiple tests need to be used to detect the dryness [6,7]. Various tests can be used to assess the ocular tear film and tear quality and quantity, such as the tear ferning (TF) test [8-10], tear osmolarity measurement [11-13], tear break-up time [14-15], tear meniscus height [16], and Schirmer's test [17-18].

TF test is one of the most reliable and repeatable tests to measure the quality of the tears during the day [19-21]. Tears, when dried, produce ferns that can be seen using a light microscope. Such ferns represent the interactions between tears components, such as proteins, large molecules and electrolytes [22]. TF images are very characteristics and specific to the state of the tears. Rolando classifies TF images into four categories in which normal eyes represent by ferns of types I and II and dry eyes represent by types III and IV [9]. Type I pattern shows ferns that are highly packed, while, type IV shows poor or no patterns at all [9]. Such grading scale has good repeatability and utility [23-24]. But, it is difficult to differentiate between TF of types I and II. A new TF grading scale that consists of five grades, $0,1,2,3$ and 4 has been recently developed [8] that can easily differentiate between different types of ferns.

Recently, we reported the first step towards the automatic diagnosis of dry eye, based on the five-point grading scale, using computer-based image analysis of tears ferning features [25]. Its objective was to characterize each reference image by a vector of characteristics. It proposed 5 vectors (of 8 components each), characteristic of each reference image and significantly different from the others. The selection of characteristics of the images was based on 3 different spaces known as texture analysis, power frequency and linear features. Noise reduction and contrast enhancement were performed for each image as a preprocessing step. A scale that has the same order of magnitude, in one characteristic vector and weights that assigned to each component was chosen according to the importance of each component. The current work is directed towards the establishment of a computer 
aided automatic classification of DE using the vector characteristic of TF images. Recently an automated identification of normal and dry eye using infrared thermography images has been developed [26], which provides only 2 classes of grading as normal and DE. In our proposed method 5 classes of DE are provided.

\section{Methods and Materials}

Ethical approval was obtained from the College of Applied Medical Sciences Ethics Committee, King Saud University. The TF images for 100 subjects (20-26 years old) were used as a sample to test the proposed computer-based classification method. A tear sample $(1 \mu \mathrm{l})$ was collected from the lower meniscus from either the right or left eye using a glass capillary tube $(10 \mu \mathrm{l}$, Sigma-Aldrich Chemical Company, UK). The tear was dried on a glass slide at room temperature $\left(23^{\circ} \mathrm{C}\right.$; humidity $\left.<45 \%\right)$. The tear ferns formed were seen using a digital microscope (Olympus DP72, Tokyo, Japan; 10× magnification) and were graded by two experts using the five-point TF grading scale [8]. TF grades higher than 2 were considered as a dry eye [8]. The processing of TF images was carried out using MATLAB R2016a Image Processing Toolbox (MathWorks Company, Natick, Massachusetts 01760 USA) and "See5" tools for the data mining (Data Mining Tools See5 and C5.0).

A preprocessing step is first performed to filter the images, enhance their contrast and normalize the gray levels by a histogram equalization method. Then, a vector characteristic is extracted from each image to represent it in the classification process. The vector characteristic used in this project combines features from texture analysis, linear structure analysis in time domain and power spectrum analysis in Fourie space, developed in detail earlier [25]. Co-occurrence matrix-based parameters [26,27], including angular second moment, contrast, correlation, entropy. The vector characteristic is represented in Eq. (1) [25].

$V C=\left[\begin{array}{l}P S(19), P S(80), P S(190), N B L, \\ A G M 0, A G M 45, C O R R 0, C O N 0, C O N 45\end{array}\right]$

Where, PS(19) is the power spectrum at the low frequency $(19 \mathrm{~Hz})$ in the radial plot, PS(80) is the power spectrum at the medium frequency $(80 \mathrm{~Hz})$ in the radial plot, PS(190) is the power spectrum at the high frequency at pixel 190 in the radial plot, NBL is the number of line detected in the image, AGM0 is the angular moment at direction $0^{\circ}, \mathrm{AGM} 45$ is the angular moment at direction $45^{\circ}$, CORR 0 is the correlation at direction $0^{\circ}, \mathrm{CON} 0$ is the contrast at direction $0^{\circ}$ and CON45 is the contrast at direction $45^{\circ}$. Analysis of feature relevance was made among all the textural features, and only 4 statistical measures out of the 14 co-occurrence features defined by Haralick has been proven to be relevant to the classification TF images [25]. The vector characteristic was split into three parts to provide different weights for components obtained from different sources [Eq. (2)].

$$
V C=\alpha V C_{P S}+\beta V C_{L}+\gamma V C_{T A}
$$

Where, VCPS is the first three components related to the normalized power spectrum, VCL includes the normalized number of lines and VCTA includes the last five components related to the texture analysis. $\alpha, \beta$ and $\gamma$ are weights that were given to each of the three sub-vectors to balance their contributions. After the experimentaltesting, the optimal values of the weights were $\alpha=35 \%$, $\beta=15 \%$ and $\gamma=50 \%$. The details about the vector characteristics development and normalization were previously discussed [25]. As described before, the classification is based on a combination of several types of characteristics (co-occurrence matrix-based features, power spectrum features and linear components) in the images. Five VCs were calculated first for the reference TF images of the five-point grading scale [8]. Those VCs are considered as references for each of the five points grading scale. For each the $100 \mathrm{TF}$ images a VC was first constructed than compared to the 5 references VCs using cluster analysis and decision tree methods.

The classification of subjects' TF images, using the proposed vector characteristic was based on a decision tree approach and K-mean classifier. The use of decision tree approach for the classification was chosen for several reasons. The tree can be easily accessible and interpreted and it is computationally efficient. Also, it provides a good classification of satellite images having analogous features to TF images [28-30]. The data mining tools See5/C5 are sophisticated for discovering patterns that delineate categories, assembling them into classifiers, and using them to make predictions. See5 generates decision trees and allow different weight to VC components. It was used to classify and automatically grade the TF images, a comparison with K-mean classifier using Euclidian distance has been established on the $100 \mathrm{TF}$ images.

\section{Results}

Table 1: The automatic grading vs the manual grading $(\mathrm{N}=100)^{\mathrm{a}-\mathrm{c}}$

\begin{tabular}{|c|c|c|c|c|c|c|}
\hline Manual Grading & Samples & $\begin{array}{c}\text { Automatic } \\
\text { Grading }\end{array}$ & & & & \\
\hline & & 0 & 1 & 2 & 3 & 4 \\
\hline 0 & 18 & 15 & 2 & 1 & 0 & 0 \\
\hline 1 & 52 & 2 & 45 & 3 & 2 & 0 \\
\hline 2 & 12 & 1 & 2 & 8 & 0 & 1 \\
\hline 3 & 10 & 0 & 1 & 1 & 7 & 1 \\
\hline 4 & 8 & 0 & 1 & 0 & 1 & 6 \\
\hline
\end{tabular}


One hundred TF images were used in this study (Figures 1 \& 2) show TF images for some normal and dry eye subjects, respectively. Five characteristics vectors were constructed as references of the 5 grades. Each vector represents one grade of the five-point grading scale [8]. Each new TF image was entered to the software. First, a vector characteristic was constructed to each TF image of the 100 test images used in our experiment. Then, a decision tree classifier procedure was run using the by See5 software. At the end of the execution, each TF image was assigned automatically to one of the five grades (0-4). To check the accuracy of the result, two experts established a manual classification of the $100 \mathrm{TF}$ images, and each image was assigned manually to one of the five grades (0-4). The results of the automatic and manual grading of TF images are represented in Table 1.
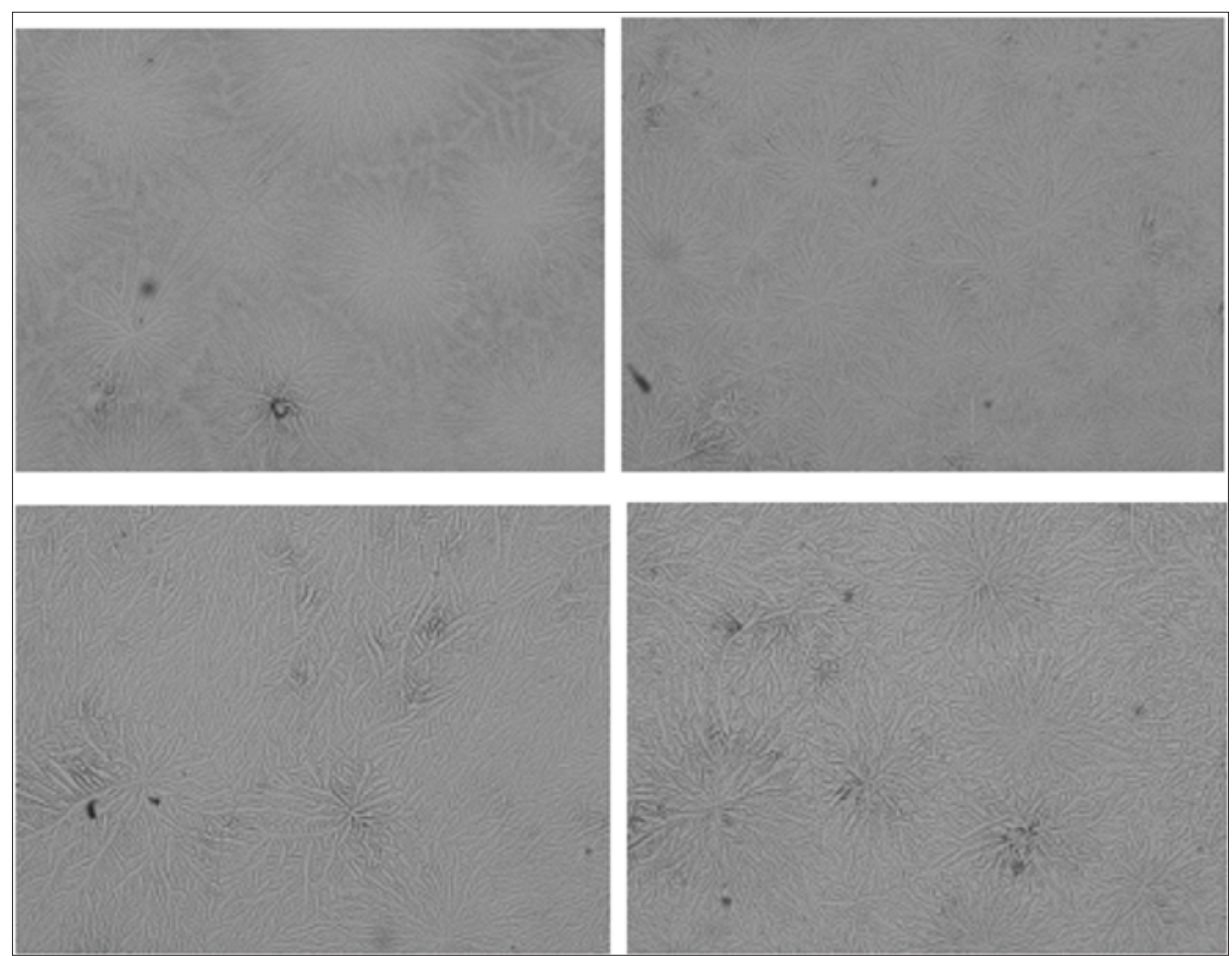

Figure 1: TF images for some normal eye subjects.
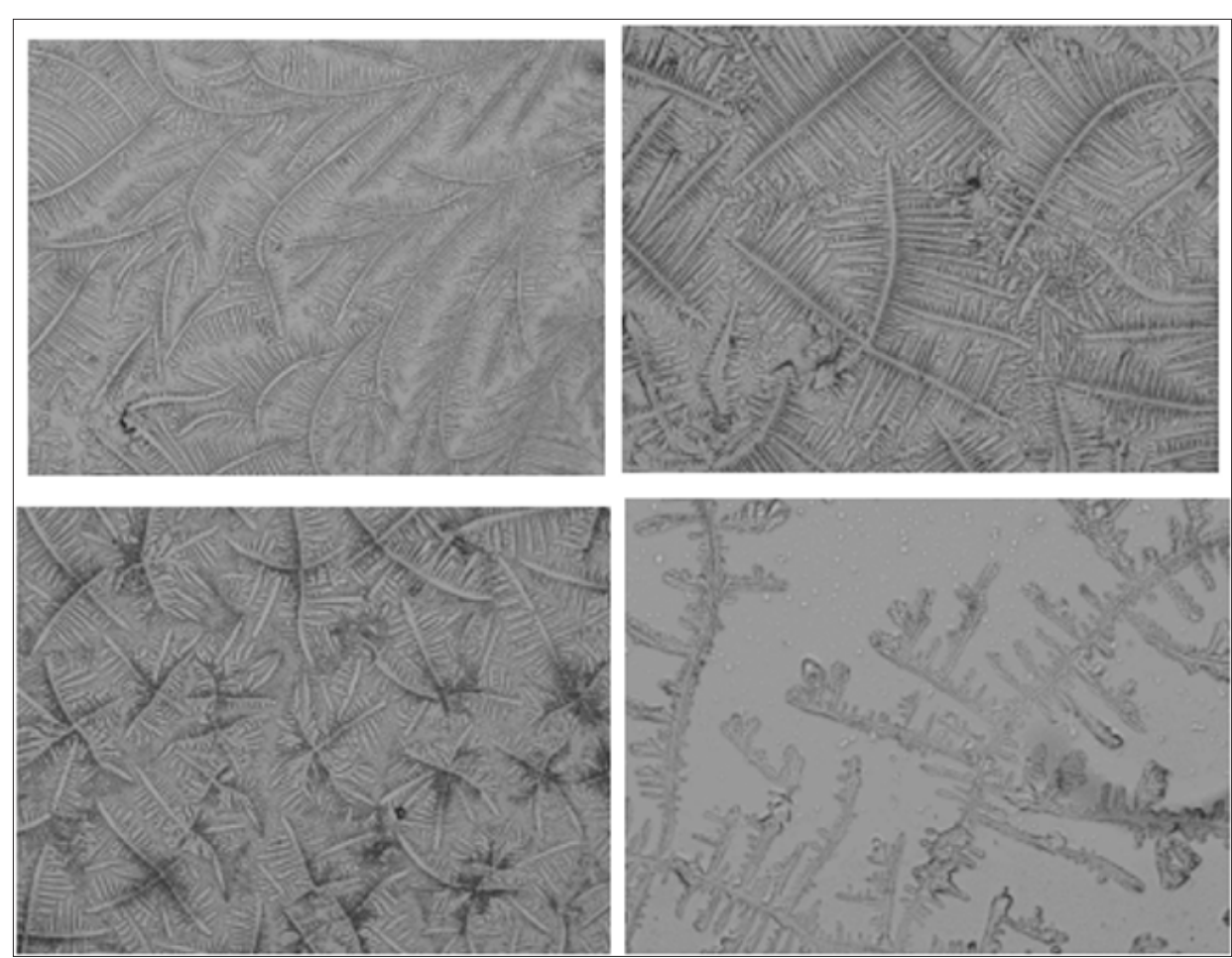

Figure 2: TF images for some dry eye subjects. 
The percentage of the perfect matching between the manual and the automatic grading was $81 \%$. The mismatching by one grade difference between the automatic and the manual grading was $12 \%$. The mismatching by at least two grades differences was found to be $7 \%$ (Table 1). The K-mean classifier was excluded due to the poor results obtained (only $61 \%$ match the manual classification).

a) The dark grey boxes represent the automatic classification that exactly match the manual ones.

b) The light grey boxes represent the misclassification by one grade difference between the automatic and the manual grading.

c) The white boxes represent a complete misclassification in which the difference between the automatic and manual grading was a least two grades difference.

\section{Discussion}

The natural tear ferning images are not limited to the 5-grade point, most TF images are located between grades and could be closer to one grade than the other. A real classification of TF images could be finer than the 5 points Masmali grading scale, but here we used a normal approximation of the TF images. For example, if the TF image is between grade 2 and 3 and it has a real grading of 2.6 it was assigned to grade 3 . Clearly, a high degree of classification overlap $(12 \%)$ was observed in the case of neighbor grades (differences by one grade). However, the proposed vector characteristic combined with the decision tree method of classification cannot perfectly distinguish between the neighbor classes. This is due to two issues, the first one is the TF images used are for real patient tear (not synthetic) and the grading is not exactly an integer number from 0 to 4 but, could be a real number, for example 1.2. The second issue is related to the vector characteristic itself since it has a limited capacity to perfectly distinguish between very similar TF images. Future work is still needed to improve the accuracy of the classification. Further development of the VC by introducing more features is also needed. Moreover, the development of database of synthetic tears TF images with known grading is primordial for calibrating the classification result.

\section{Conclusion}

The proposed computer based automatic classification for dry eye using tear ferning images is the first of its kind up-todate, it provides a pretty good classification result, an overall accuracy of $81 \%$ without any human intervention. The result was compared to the manual grading of two experts. The percentage of misclassification between the manual and automatic grades was found to be $7 \%$ (at least two grades difference) and $12 \%$ (one grade difference). An improvement of the vector characteristic is still needed to increase the accuracy of the automatic grading of TF images. Future research should also involve a database of synthetic tears TF grades, to be used in the automatic DE classification.

\section{Acknowledgement}

The authors extend their appreciation to the College of Applied Medical Sciences Research Centre and the Deanship of Scientific Research at King Saud University for funding this research, and to thank Dr. Gamal El-Hiti for beneficial comments and fruitful discussions.

\section{References}

1. Farandos NM, Yetisen AK, Monteiro MJ, Lowe CR, Yun SH (2015) Contact lens sensors in ocular diagnostics. Adv Healthcare Mater 4(6): 792-810.

2. Tiffany JM (2003) Tears in health and disease. Eye 17(8): 923-926.

3. Szczesna DH, Jaroński J, Kasprzak HT, Stenevi U (2006) Interferometric measurements of dynamic changes of tear film. J Biomed Opt 11(3): 34028.

4. Johnson ME, Murphy PJ (2004) Changes in the tear film and ocular surface film from dry eye syndrome. Prog Retin Eye Res 23(4): 449-474.

5. (2007) The definition and classification of dry eye disease: report of the definition and classification subcommittee of the International Dry Eye WorkShop 2007. Ocul Surf 5(2): 75-92.

6. Kent C (2013) Dry eye diagnosis: $21^{\text {st }}$-Century tools. Rev Ophthalmol 13 : 28-41.

7. Savini G, Prabhawasat P, Kojima T, Grueterich M, Espana E, et al. (2008) The challenge of dry eye diagnosis. Clin Ophthalmol 2(1): 31-55.

8. Masmali AM, Murphy PJ, Purslow C (2014) Development of a new grading scale for tear ferning. Cont Lens Anterior Eye 37(3): 178-184.

9. Rolando M (1984) Tear mucus ferning test in normal and keratoconjunctivitis sicca eyes. Chibret Int J Ophthalmol 2(4): 32-41.

10. Maragou M, Vaikousis E, Ntre A, Koronis N, Georgiou P, et al. (1996) Tear and saliva ferning tests in Sjogren's syndrome (SS). Clin Rheumatol 15(2): 125-132.

11. Masmali A, Alrabiah S, Alharbi A, El Hiti GA, Almubrad T (2014) Investigation of tear osmolarity using the TearLabTM osmolarity system in normal adults in Saudi Arabia. Eye Contact Lens 40(2): 74-78.

12. Szalai E, Berta A, Szekanecz Z, Szûcs G, Módis L (2012) Evaluation of tear osmolarity in non-Sjögren and Sjögren syndrome dry eye patients with the TearLab system. Cornea 31(8): 867-871.

13. Lemp MA, Bron AJ, Baudouin C, Benítez Del Castilo JM, Geffen D, et al. (2011) Tear osmolarity in the diagnosis and management of dry eye disease. Am J Ophthalmol 151(5): 792-799.

14. Kallarackal GU, Ansari EA, Amos N, Martin JC, Lane C, et al. (2008) A comparative study to assess the clinical use of fluorescein meniscus time (FMT) with tear break up time (TBUT) and Schirmer's tests (ST) in the diagnosis of dry eyes. Eye 16(5): 594-600.

15. Lee JH, Kee CW (1988) The significance of tear film break-up time in the diagnosis of dry eye syndrome. Korean J Ophthalmol 2(2): 69-71.

16. Tiffany JM (2006) Surface tension in tears. Arch Soc Esp Oftalmol 81(7): 363-366.

17. Masmali A, Alqahtani TA, Alharbi A, El Hiti GA (2014) Comparative study of repeatability of phenol red thread test versus Schirmer's test in normal adults in Saudi Arabia. Eye Contact Lens 40(3): 127-131.

18. Rose JE, Roepke MH (1957) An acidified antigen for detection of nonspecific reactions in the plate-agglutination test for bovine brucellosis. Am J Vet Res 18(68): 550-555.

19. Masmali AM, Al Bahlal JM, El Hiti GA, Akhtar S, Purslow C (2015) Repeatability and diurnal variation of tear ferning test. Eye Contact Lens 41(5): 262-267.

20. Masmali AM, Al Qhtani S, Al Gasham TM, El Hiti GA, Purslow C, et al. (2013) Application of a new grading scale for tear ferning in non-dry eye 
and dry eye subjects. Cont Lens Anterior Eye 38(1): 39-43.

21. Masmali AM, Al Shehri A, Alanazi SA, Abusharaha A, Fagehi R, El Hiti GA (2016) Assessment of tear film quality among smokers using tear ferning patterns. J Ophthalmol.

22. Masmali A, Purslow C, Murphy PJ (2014) The tear ferning test: a simple clinical technique to evaluate the ocular tear film. Clin Exp Optom 97(5): 399-406.

23. Pensyl CD, Dillehay SM (1998) The repeatability of tear mucus ferning grading. Optom Vis Sci 75(8): 600-604.

24. Srinivasan S, Joyce E, Jones LW (2007) Tear osmolarity and ferning patterns in postmenopausal women. Optom Vis Sci 84(7): 588-592.

25. Saad AS, El Hiti GA, Masmali AM (2015) A computer-based image analysis for tear ferning featuring. J Innov Opt Heal Sci 8(5): 1-10.

ISSN: 2574-1241

DOI: 10.26717/BJSTR.2018.08.001702

Ali S Saad. Biomed J Sci \& Tech Res

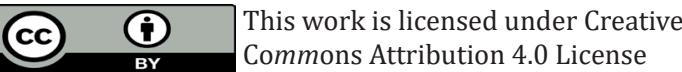

Submission Link: https://biomedres.us/submit-manuscript.php
26. Haralick R (1979) Statistical and Structural Approaches to Texture. IEEE Proceedings 67(5): 786-804.

27. Walker RF, Jackway PT, Longstaff ID (1997) Recent Developments in the Use of the Co-occurrence Matrix for Texture Recognition. In $13^{\text {th }}$ International Conference on Digital Signal Processing Proceedings 1: 63-65.

28. Koperski K, Marchisio G, Tusk C, Aksoy S (2002) Interactive models for semantic labeling of satellite images. In Proc. International Symposium on Optical Science and Technology SPIE's $47^{\text {th }}$ Annual Meeting, Seattle.

29. Tusk C, Koperski K, Aksoy S, Marchisio G (2003) Automated Feature Selection through Relevance Feedback. In Geoscience and Remote Sensing Symposium, IGARSS'03, Proceedings 2003 IEEE International 6: 3691-3693.

$\begin{array}{ll}\text { BIOMEDICAL } & \text { Assets of Publishing with us } \\ \text { RESEARCHES } & \text { - Global archiving of articles } \\ \text { - Immediate, unrestricted online access } \\ \text { - RsN: } 2574-1241\end{array}$

